\title{
DINÂMICA FOLICULAR E TAXA DE CONCEPÇÃO DE VACAS NELORE SINCRONIZADAS COM DIFERENTES PROTOCOLOS DE INDUÇÃO DA OVULAÇÃO
}

\author{
Priscila Assis Ferraz¹, Alexandra Soares Rodrigues ${ }^{1}$, Marcus Vinícius Galvão \\ Loiola1, Marcos Chalhoub Coelho Lima1, Thereza Cristina Borio dos Santos Calmon \\ Bittencourt ${ }^{1}$, Antonio de Lisboa Ribeiro Filho'
}

1 UFBA

Correspondência: Priscila Assis Ferraz: prisferraz@gmail.com

\begin{abstract}
RESUMO: Objetivou-se avaliar a dinâmica folicular e a taxa de concepção em vacas Nelore sincronizadas com diferentes indutores da ovulação. Em um dia aleatório do ciclo estral (D0), 196 fêmeas receberam um dispositivo intravaginal de progesterona (P4) e uma injeção de $2 \mathrm{mg}$ de Benzoato de estradiol (BE) im. No D8, retirou-se o dispositivo de P4 e foram administrados $150 \mu \mathrm{g}$ de d-Cloprostenol e $10 \mathrm{mg}$ de FSHp im. Neste momento, os animais foram distribuídos em três grupos de acordo com o indutor da ovulação: Grupo $B E(n=62)$ - os animais foram tratados com 1 mg de BE im no D8; Grupo GnRH ( $n=62)$ - os animais receberam $25 \mu \mathrm{g}$ de Acetato de Gonadorelina $(\mathrm{GnRH})$ im no D10 e o Grupo BE+GnRH ( $\mathrm{n}=72)$ - os animais foram tratados com $1 \mathrm{mg}$ de BE no D8 e $25 \mu \mathrm{g}$ de GnRH no D10. Os animais foram inseminados 48h após a retirada do dispositivo de P4 (D10) e o diagnóstico de gestação foi realizado 30 dias após a IATF. Para avaliação da dinâmica folicular foram utilizadas 10 fêmeas de cada grupo e as ultrassonografias foram realizadas a cada $8 \mathrm{~h}$ a partir do D8 até o momento da ovulação (MOV). A análise estatística foi realizada pelo SPSS, versão 19. A média do DFOL para grupo $\mathrm{GnRH}(13,19 \pm 1,43 \mathrm{~mm})$ foi significativamente superior aos

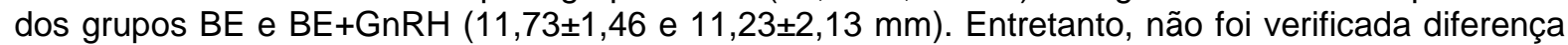
significativa para a taxa de concepção, que foi de 59,$7 ; 61,3 ; 54,2 \%$, respectivamente, para os grupos $\mathrm{BE}, \mathrm{GnRH}$ e $\mathrm{BE}+\mathrm{GnRH}$. Os fármacos utilizados, assim como, sua associação resultaram em uma eficiente resposta ovariana e satisfatória taxa de concepção, entretanto, a associação entre o $\mathrm{BE}$ e o GnRH não promoveu uma melhora desses índices.
\end{abstract}

Palavras-chave: vacas Nelore; IATF; ovulação; BE e GnRH

\section{FOLLICULAR DYNAMICS AND CONCEPTION RATE OF NELORE COWS SYNCHRONIZED WITH DIFFERENT PROTOCOLS OF OVULATION INDUCTION}

\begin{abstract}
This study aimed to evaluate the follicular dynamics and conception rate in Nellore cows synchronized with different inducing ovulation. On a random day of the estrous cycle (D0), 196 females received an intravaginal progesterone device (P4) and an injection of $2 \mathrm{mg}$ of estradiol benzoate (EB) im. At D8 withdrew the device P4 and were given $150 \mu \mathrm{g}$ of $\mathrm{d}$-cloprostenol and $10 \mathrm{mg}$ im FSHp. At this time, the animals were divided into three groups according to the ovulation inducer: BE Group $(n=62)$ - animals were treated with $1 \mathrm{mg}$ of EB im on D8; GnRH group $(n=62)$ - the animals received $25 \mu \mathrm{g}$ Acetate gonadorelin $(\mathrm{GnRH})$ im in BE group and D10 $+\mathrm{GnRH}(n=72)$ animals were treated with $1 \mathrm{mg}$ of EB on D8 and D10 in $25 \mu \mathrm{g} \mathrm{GnRH}$. The animals were inseminated $48 \mathrm{~h}$ after removal device of P4 (D10) and pregnancy diagnosis was performed 30 days after FTAl. For evaluation of follicular dynamics were used 10 females from each group and ultrasounds were performed every 8h from D8 to the time of ovulation (MOV). Statistical analysis was performed using SPSS, version 19. The average DFOL to $\mathrm{GnRH}$ group $(13.19 \pm 1.43 \mathrm{~mm})$ was significantly higher than in groups $B E$ and $B E+G n R H(11.73 \pm 1.46$ and $11.23 \pm 2.13 \mathrm{~mm})$. However, there was no significant difference in conception rate, which was $59.7,61.3,54.2 \%$, respectively, for groups $\mathrm{BE}, \mathrm{BE}$ $+\mathrm{GnRH}$ and GnRH. The drugs used, as well as their association resulted in an efficient ovarian response and satisfactory conception rate, however, the association between $\mathrm{BE}$ and GnRH did not promote an improvement in these indexes.
\end{abstract}

Key Words: Nelore cows; IATF; ovulation, BE and GnRH 


\section{INTRODUÇÃO}

Diversos protocolos hormonais têm sido desenvolvidos com a finalidade de induzir e sincronizar o estro e a ovulação, possibilitando assim, a utilização da Inseminação Artificial em Tempo Fixo (IATF; Barros e Enero, 2004). Esta biotecnologia permite que os animais sejam inseminados sem a necessidade de detecção de estro e em horário pré-determinado, promovendo maior disseminação da inseminação artificial (Inforzato et al., 2008).

A associação dos estrógenos e seus ésteres em protocolos a base de progesterona (P4) é um dos tratamentos mais utilizados para sincronização da onda de crescimento folicular em fêmeas bovinas (Macmillan e Burke, 1996). A administração de prostaglandina $\mathrm{F} 2 \alpha$ (PGF2 $\alpha$ ) no momento da retirada do dispositivo de P4 proporciona o término da fase luteínica de forma sincronizada (Odde, 1990). E a ovulação pode ser induzida com o emprego de fármacos como a Gonadotrofina Coriônica humana (hCG), o Hormônio Luteinizante (LH), o Hormônio Liberador de Gonadotrofina (GnRH) e os estrógenos e seus ésteres (Bó et al., 2003).

Para a realização da IATF é importante conhecer o momento da ovulação (Binelli, Ibiapina e Bisinoto, 2006). De acordo com Meneghetti et al. (2009), o intervalo entre a aplicação dos indutores e a ovulação pode variar conforme o fármaco utilizado. Além disso, a assincronia da ovulação e a grande variabilidade da resposta aos tratamentos hormonais são alguns dos fatores que podem comprometer 0 sucesso dos programas de IATF (Mizuta, 2003). Assim, estratégias têm sido desenvolvidas para melhorar a resposta e a eficiência destes protocolos, buscando uma ovulação de forma mais sincrônica e melhores índices reprodutivos (Sá filho et al., 2007).

Neste contexto, a aplicação de estrógeno como indutor da ovulação no momento da retirada do dispositivo intravaginal de P4 (Cavalieri et al., 2002) ou do GnRH no momento da IATF como estímulo ovulatório, são alternativas para reduzir o número de manejos dos animais e melhorar a eficiência dos protocolos de sincronização (Martinez et al., 2001). Além disso, a associação de indutores da ovulação pode ser empregada visando uma melhor sincronia das ovulações e melhora na resposta aos tratamentos hormonais (Baruselli et al, 2007).

O presente estudo foi realizado com o objetivo de avaliar a dinâmica folicular e a taxa de concepção de vacas Nelore submetidas a um protocolo de sincronização do crescimento folicular, com a indução das ovulações por Benzoato de Estradiol, GnRH ou a associação entre os mesmos e posterior IATF.

\section{MATERIAL E MÉTODOS}

O experimento foi conduzido em uma fazenda comercial de gado de corte localizada no Centro Sul do Estado da Bahia. Esta propriedade está inserida numa região do semiárido e possui latitude de $13^{\circ} 26^{\prime} 28^{\prime \prime}$ Sul e longitude de $40^{\circ} 25^{\prime} 51^{\prime \prime}$ Oeste, os dados foram coletados no período de abril a maio de 2011.

Foram utilizadas 196 fêmeas bovinas multíparas da raça Nelore, com o intervalo pós-parto de $96,04 \pm 20,70$ dias, idade de $5,71 \pm 1,52$ anos e escore de condição corporal (ECC) de $3,02 \pm 0,31$ (escala de 1 a 5; Houghton et al., 1990). Os animais foram mantidos em pasto de capim Buffel (Cenchrus ciliares) com água e mineral ad libitum.

Previamente ao início do protocolo de sincronização do crescimento folicular e ovulação, esses animais 
foram submetidos ao exame clínicoginecológico e ultrassonografia transretal, utilizando-se um transdutor linear com frequência de 6,0 MHz (PieMedical, Falco 100, Maastricht, Netherlands), sendo considerados aptos a participarem do experimento os animais que não tinham histórico de aborto e apresentaram no momento da avaliação ausência de anormalidades do trato reprodutivo.

As fêmeas foram submetidas ao seguinte protocolo de sincronização: em dia aleatório do ciclo estral, denominado dia 0 (D0), foi inserido um dispositivo intravaginal de progesterona (P4; PRIMER $^{\circledR}$, Tecnopec, São Paulo, Brasil) e administrado por via intramuscular (im) $2 \mathrm{mg}$ de Benzoato de Estradiol (RIC-BE ${ }^{\circledR}$, Tecnopec, São Paulo, Brasil. No D8, retirou-se a $\mathrm{P} 4$ e foram administrados $150 \mu \mathrm{g}$ de d-Cloprostenol (PROLISE $^{\circledR}$, Tecnopec, São Paulo, Brasil) im. e $10 \mathrm{mg}$ de FSHp (FOLLTROPIN $^{\circledR}$, Tecnopec, São Paulo, Brasil) im. Neste dia, os animais foram divididos homogeneamente de acordo com idade, escore de condição corporal e intervalo pós-parto em três grupos experimentais conforme o protocolo de indução da ovulação: Grupo BE ( $n=62)$ - os animais foram tratados com $1 \mathrm{mg}$ de Benzoato de Estradiol (RIC-BE ${ }^{\circledR}$, Tecnopec, São Paulo, Brasil) im no D8; Grupo GnRH (n=62) - os animais receberam $25 \quad \mu g$ de Lecirelina (GESTRAN $^{\circledR}$, Tecnopec, São Paulo, Brasil) im no D10 e o Grupo BE+GnRH $(n=72)$ - os animais foram tratados com $1 \mathrm{mg}$ de Benzoato de Estradiol no D8 e $25 \mu \mathrm{g}$ de Lecirelina no D10. Todas as intervenções foram iniciadas no mesmo horário, ás 8:00 h, conforme Figura 1.
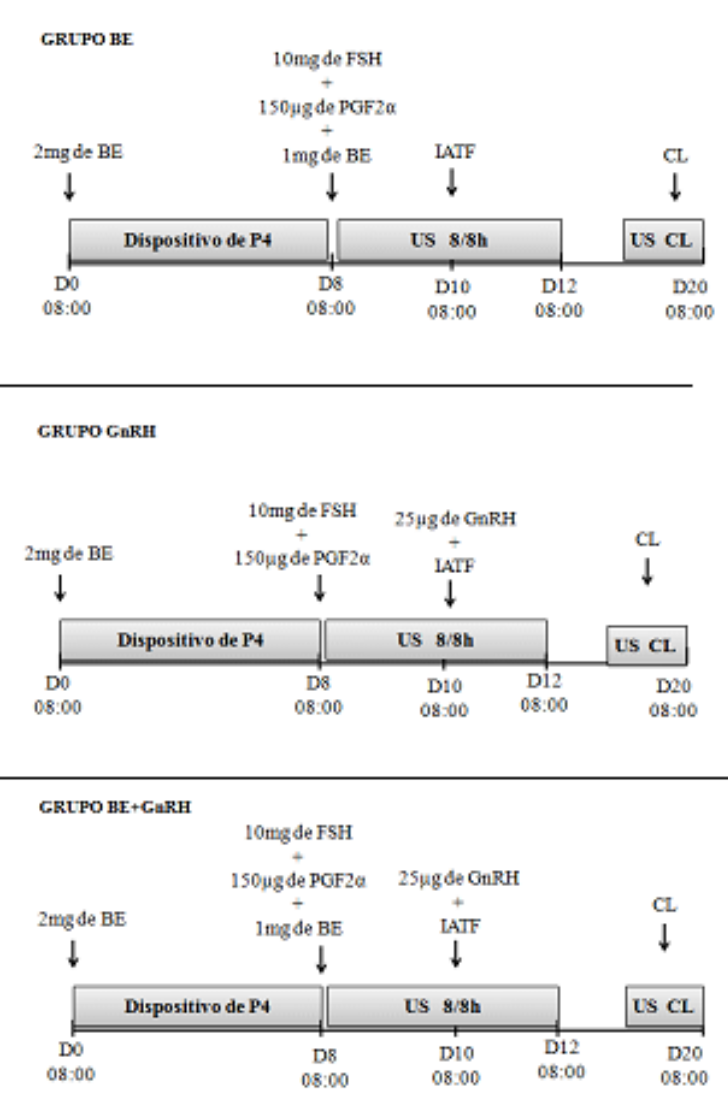

Figura 1 - Representação esquemática dos diferentes protocolos de indução da ovulação e dos intervalos das avaliações ultrassonográficas (US) em vacas Nelore.

Para avaliação da dinâmica folicular utilizou-se 10 fêmeas de cada grupo experimental. No D8 do protocolo foi iniciada a mensuração folicular por ultrassonografia transretal utilizando-se um transdutor linear de $6,0 \mathrm{MHz}$ (PieMedical, Falco 100, Maastricht, Netherlands) a cada $8 \mathrm{~h}$ até o momento da ovulação (MOV) ou 96 horas após a retirada do dispositivo intravaginal de progesterona. A taxa de crescimento do folículo ovulatório (TXFOL) foi obtida pela diferença entre o diâmetro do folículo ovulatório (DFOL) e o diâmetro do maior folículo na primeira avaliação no D8 (FOLD8), dividido pelo período de crescimento e expresso na unidade de milímetros por dia $(\mathrm{mm} / \mathrm{d})$. Outro exame ultrassonográfico foi realizado em todos os animais no D20 para confirmação da ovulação e mensuração do diâmetro do corpo lúteo (DCL). A ovulação foi definida pelo desaparecimento do 

protocolos de indução da ovulação

folículo ovulatório presente no exame anterior com consequente formação do corpo lúteo. Todas as mensurações foram efetuadas por um mesmo técnico.

Todos os 196 animais foram inseminados $48 \mathrm{~h}$ após a retirada da P4 (D10) por um mesmo técnico utilizandose doses de sêmen criopreservado de um mesmo touro da raça Nelore e pertencente a uma única partida, as doses foram previamente avaliadas seguindo os requisitos do Colégio Brasileiro de Reprodução Animal (Henry e Neves, 1998). No momento da inseminação, o sêmen foi descongelado a $37^{\circ} \mathrm{C}$ por 30 segundos.

O diagnóstico de gestação foi realizado 30 dias após a IATF por meio da ultrassonografia transretal utilizandose um transdutor linear de $6,0 \mathrm{MHz}$ (PieMedical, Falcon 100, Maastricht, Netherlands) e foi considerado como diagnóstico positivo de gestação a presença de uma vesícula embrionária com um embrião viável (presença de batimento cardíaco). A taxa de concepção foi calculada pela proporção de vacas gestantes sobre o total de vacas inseminadas.

Para a análise estatística dos dados foi utilizado o Statistical Package for Social Science (SPSS, versão 19) considerando nível de significância de $5 \%$ e atendendo os padrões de amostragem e graus de liberdade estabelecidos por Sampaio (1998). Para a análise das variáveis: diâmetro do maior folículo na primeira avaliação (FOLD8), diâmetro do folículo ovulatório (DFOL), momento da ovulação (MOV) e diâmetro do corpo lúteo (DCL) foi utilizado o teste Kruskal-Wallis. A taxa de crescimento do folículo ovulatório (TXFOL) foi comparada pelo teste Wilcoxon-Ranks. Além disso, foi quantificada a associação entre as variáveis DFOL e DCL pelo coeficiente de correlação de Spearman. Para comparação da taxa de concepção entre os grupos experimentais foi empregado o teste do Qui-quadrado $\left(\chi^{2}\right)$.

\section{RESULTADOS E DISCUSSÃO}

\section{Diâmetro folicular}

A média geral para o DFOL foi de $12,07 \pm 1,83 \mathrm{~mm}$. Não houve diferença significativa na comparação entre 0 FOLD8 e os grupos experimentais BE, $\mathrm{GnRH}$ e BE + GnRH, respectivamente $9,62 \pm 0,93 ; 9,15 \pm 1,59$ e $8,43 \pm 2,60 \mathrm{~mm}$. As médias encontradas para o DFOL entre os grupos BE, $\mathrm{GnRH}$ e $\mathrm{BE}+\mathrm{GnRH}$ foram respectivamente, $11,73 \pm 1,46$; $13,19 \pm 1,43$ e $11,23 \pm 2,13 \mathrm{~mm}$. $\mathrm{Na}$ comparação entre as médias do DFOL observou-se que o grupo $\mathrm{GnRH}$ obteve uma média para o DFOL superior aos grupos $\mathrm{BE} \quad(\mathrm{P}=0,04)$ e $\mathrm{BE}+\mathrm{GnRH}$ $(P=0,03 ;$ Tabela 1).

\begin{tabular}{|c|c|c|c|c|c|}
\hline Variávers $_{\text {Tratamentos }}^{\text {Tres }}$ & $B E(n=10)$ & $\operatorname{GnRH}(n=10)$ & $B E+G n R H(n=9)$ & Total & P-valor \\
\hline $\begin{array}{l}\text { FOLD8 (mm) } \\
\text { (m) }\end{array}$ & $9,62 \pm 0,93$ & $9,15 \pm 1,59$ & $8,43 \pm 2,60$ & $9,06 \pm 1,80$ & 0,46 \\
\hline $\mathrm{DFOL}(\mathrm{mm})$ & $11,73 \pm 1,46^{\circ}$ & $13,19 \pm 1,43^{\mathrm{a}}$ & $11,23 \pm 2,13^{\circ}$ & $12,08 \pm 1,83$ & 0,04 \\
\hline $\begin{array}{c}\text { TXFOL } \\
\text { (mm/dia) }\end{array}$ & $0,58 \pm 0,34^{\circ}$ & $1,07 \pm 0,34^{\mathrm{a}}$ & $0,47 \pm 0,40^{\circ}$ & $0,72 \pm 0,43$ & 0,0003 \\
\hline MOV (h) & $60,72 \pm 14,10$ & $72,17 \pm 13,71$ & $68,59 \pm 12,81$ & $67,11 \pm 13,98$ & 0,19 \\
\hline $\mathrm{DCL}(\mathrm{mm})$ & $19,08 \pm 2,95$ & $16,65 \pm 1,95$ & $18,44 \pm 3,77$ & $18,04 \pm 3,04$ & 0,14 \\
\hline
\end{tabular}

Os resultados desta pesquisa foram semelhantes aos relatados por Crepaldi (2009) e Reis et al. (2004), os quais obtiveram diâmetro médio do folículo ovulatório de $12,00 \mathrm{~mm}$ em vacas Nelore quando utilizaram Benzoato de Estradiol como indutor da ovulação no momento da retirada do dispositivo de progesterona em um protocolo para IATF. Similarmente, Cavalieri et al. (2002) utilizando o Benzoato de Estradiol neste momento do protocolo encontraram média para o diâmetro do folículo ovulatório de $11,10 \pm 0,40 \mathrm{~mm}$.

Entretanto, Ross et al. (2004) encontraram média para o diâmetro do folículo ovulatório de 10,90 $\pm 0,50 \mathrm{~mm}$ numericamente inferior aos relatados nesta pesquisa para o Benzoato de 
Estradiol administrado no momento da retirada da fonte de progesterona. Esses autores afirmam que quando o Benzoato de Estradiol é administrado no momento da retirada da fonte progesterona a concentração deste hormônio ainda encontra-se elevada ocorrendo menor crescimento folicular e consequentemente menor folículo ovulatório.

Os resultados encontrados neste estudo para o DFOL no grupo GnRH corroboram aos achados de Murta (2011) e Thompson et al. (1999) quando aplicaram o $\mathrm{GnRH} 48 \mathrm{~h}$ após a retirada da progesterona e verificaram média de diâmetro do folículo ovulatório de respectivamente, $13,50 \pm 0,29 \mathrm{~mm}$ e $13,70 \pm 0,80 \mathrm{~mm}$ para vacas Nelore.

$\mathrm{Na}$ associação entre o benzoato de estradiol e $\mathrm{GnRH}$, os resultados do DFOL obtidos no presente estudo foram similares aos encontrados por Torres Junior et al. (2007) de 11,70 $\pm 0,10 \mathrm{~mm}$, apesar de nesta associação os autores terem utilizado o Cipionato de Estradiol como éster de estradiol para indução da ovulação, demonstrando assim que o protocolo de indução da ovulação a base apenas de GnRH apresentou-se como mais eficiente em promover um maior DFOL.

Taxa de crescimento

A média geral para o TXFOL foi de $0,72 \pm 0,43 \mathrm{~mm} / \mathrm{dia}$. As médias encontradas para a TXFOL entre os grupos $\mathrm{BE}, \mathrm{GnRH}$ e $\mathrm{BE}+\mathrm{GnRH}$ foram respectivamente, $0,58 \pm 0,3 ; 1,07 \pm 0,3$ e $0,47 \pm 0,4 \mathrm{~mm} /$ dia e na comparação entre os grupos experimentais observou-se que as mesmas diferiram entre si ( $P=0,0003$; Tabela 1).

A taxa de crescimento pode justificar os resultados acima descritos em relação ao DFOL dos animais do grupo $\mathrm{GnRH}$ que foi significativamente superior aos demais protocolos de indução da ovulação, demonstrando que o tratamento com $\mathrm{GnRH}$ no momento da IA promove maior período de crescimento do folículo ovulatório (Kim et al., 2005).

Momento da ovulação

A taxa geral de ovulação foi $67,11 \pm 13,98 \mathrm{~h}$. Na comparação do momento da ovulação nos diferentes grupos experimentais não foi observada diferença estatística $(P=0,19)$. $O$ intervalo entre a retirada do dispositivo de progesterona e a ovulação foi de $60,72 \pm 14,10 ; 72,17 \pm 13,7$ e $68,59 \pm 12,8 \mathrm{~h}$, respectivamente, para os grupos $\mathrm{BE}$, $\mathrm{GnRH}$ e BE+GnRH (Tabela 1).

Resultados similares foram verificados por Crepaldi (2009) e Cutaia et al. (2001) que encontraram intervalos de, respectivamente, $58,30 \pm 2,10$ e $54,00 \pm 44,90 \mathrm{~h}$ entre a retirada do dispositivo de progesterona e a ovulação para o Benzoato de Estradiol aplicado no mesmo momento da retirada do dispositivo de progesterona em vacas de corte. Contudo, em divergência ao presente estudo, Reis et al. (2004) verificaram intervalo entre a retirada do dispositivo de progesterona e a ovulação de $68,00 \pm 1,70 \mathrm{~h}$. Apesar de não se verificar diferença significativa nesta pesquisa para o momento da ovulação entre os grupos experimentais, foi possível observar que o grupo GnRH (72,1 $\pm 13,7 \mathrm{~h})$ apresentou uma tendência numérica de um maior intervalo entre a retirada da progesterona e a ovulação quando comparado aos demais grupos.

Intervalos longos entre retirada do dispositivo de progesterona e a ovulação também foram encontrados por Sá Filho et al. (2004) quando aplicaram GnRH 54h após a retirada do implante auricular de P4 e observaram intervalo médio de $72,00 \pm 1,10 \mathrm{~h}$. Contudo, resultados divergentes foram observados por Ambrose et al. (2001), porém estes utilizaram novilhas Holandesas e observaram intervalo médio da retirada do dispositivo de progesterona e a ovulação de $56 \mathrm{~h}$ para o GnRH aplicado 48h após a retirada da progesterona. 

protocolos de indução da ovulação

Neste trabalho, os resultados para a associação do Benzoato de Estradiol com o GnRH estão de acordo com Torres-Junior et al. (2007) que combinaram o Cipionato de Estradiol na retirada do implante auricular de Norgestomet com uma aplicação de $\mathrm{GnRH} 48 \mathrm{~h}$ após a retirada do implante em vacas Nelore e verificaram média para o intervalo entre a retirada do implante e a ovulação de 72,00 $22,27 \mathrm{~h}$, demonstrando que a associação entre os indutores mantêm o momento da ovulação próximo aquele alcançado apenas com 0 emprego do $\mathrm{GnRH}$ enquanto 0 uso do $\mathrm{BE}$ promove a ocorrência de uma ovulação numericamente antecipada em relação aos demais.

\section{Diâmetro do corpo lúteo}

A média geral para o diâmetro do corpo lúteo foi de $18,04 \pm 3,03 \mathrm{~mm}$. Os diâmetros dos corpos lúteos (DCL) não diferiram entre os tratamentos $e$ as médias foram 19,08 $\pm 2,95 ; 16,65 \pm 1,95$ e $18,44 \pm 3,77 \mathrm{~mm}$, respectivamente, para os grupos BE, GnRH e BE+GnRH (Tabela 1). Além disso, não foi observada correlação entre o DFOL e o DCL ( $r=0,081 ; P=0,676)$.

As médias para o diâmetro do corpo lúteo encontradas neste estudo se assemelham as descritas por Figueiredo et al. (1997) em fêmeas Bos taurus indicus que observaram resultados de $17,69 \pm 040 \mathrm{~mm}$. Estes achados estão de acordo com os encontrados por Martin et al. (2004) que não observaram correlação entre o diâmetro do folículo ovulatório e o diâmetro do corpo lúteo e encontraram média para o diâmetro do

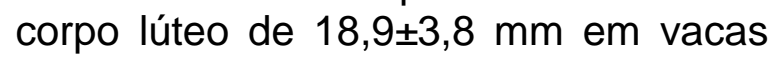
Nelore.

Entretanto, Andrade et al. (2011) estudaram a dinâmica ovulatória de vacas zebuínas utilizando um protocolo com diferentes indutores da ovulação e encontraram diâmetro médio do corpo lúteo de 17,11 mm. Além disso, esses autores verificaram uma correlação positiva e significativa entre DFOL e essas variáveis. Deste modo, verifica-se que a literatura ainda esboça uma inconsistência sobre a correlação entre - DFOL e DCL, necessitando da realização de mais estudos abordando o tema e que os três protocolos de indução da ovulação promoveram a formação de corpos lúteos com diâmetros similares.

Taxa de concepção

A taxa de concepção total foi de $58,2 \%$ e não houve diferença entre os grupos experimentais $\mathrm{BE}, \mathrm{GnRH}$ e $\mathrm{BE}+\mathrm{GnRH}$, respectivamente, 59,7\%; $61,3 \% ; 54,2 \%$, (Tabela 2).

\begin{tabular}{|c|c|c|}
\hline $\begin{array}{c}\text { Grupos } \\
\text { Experimentais }\end{array}$ & Número de animais ( $n$ ) & Taxa de concepção (\%) \\
\hline $\mathrm{BE}$ & 62 & $59,7(37 / 62)$ \\
\hline GnRH & 62 & $61,3(38 / 62)$ \\
\hline $\mathrm{BE}+\mathrm{GnRH}$ & 72 & $54,2(38 / 72)$ \\
\hline Total & 196 & $58,2(114 / 196)$ \\
\hline
\end{tabular}

Ayres et al. (2008) verificaram taxa de concepção de $58,80 \%$ para o Benzoato de Estradiol aplicado como indutor no momento da retirada do dispositivo de P4. Resultados divergentes foram encontrados por Cavalieri et al. (2002) que obtiveram taxa de concepção de $33,30 \%$ para Benzoato de Estradiol aplicado no momento da retirada do dispositivo de progesterona, entretanto estes autores utilizaram em seu estudo novilhas da raça Nelore, categoria animal que pode ter comprometido os índices de concepção obtidos na IATF.

Em relação ao grupo $\mathrm{GnRH}$, Sá Filho et al. (2004) e Ayres et al. (2006) desenvolveram um experimento semelhante ao desta pesquisa e verificaram taxas de concepção de 68,00 e $67,60 \%$, respectivamente. Contudo, ao utilizarem o mesmo protocolo em experimentos posteriores, Sá Filho et al. (2007) obtiveram taxas de concepção de 54,90\%, inferiores ao 
presente trabalho podendo ser justificado pelo fato destes autores terem utilizado animais lactantes com um período pós-parto de 30 a 60 dias, que consequentemente pode ter comprometido o crescimento folicular e a ovulação, apesar da utilização da Gonadotrofina Coriônica Equina no protocolo de sincronização.

Para a associação dos indutores da ovulação os resultados foram semelhantes aos observados por Silva et al. (2011) quando associaram Benzoato de Estradiol no momento da retirada da $\mathrm{P} 4$ e o $\mathrm{GnRH}$ no momento da IATF em vacas Nelore e encontraram taxa de concepção de 54,10\%. Porém, esses autores descrevem que a associação entre os fármacos não promoveu incremento na taxa de concepção. Da mesma forma, Crivelenti Junior, Gofert e Valentim (2012) verificaram taxa de concepção de $57,00 \%$ quando associaram BE no momento da retirada do dispositivo de P4 a aplicação de $\mathrm{GnRH}$ no momento da inseminação. Resultados contraditórios foram encontrados por Neto et al. (2012) que em um protocolo de sincronização similar verificaram uma taxa de concepção de 45,60\%, no entanto os autores acima citados utilizaram novilhas em seu modelo experimental as quais podem ter comprometido os índices reprodutivos deste programa de IATF.

Acreditava-se que a associação entre os dois indutores promoveria uma ovulação de forma mais sincronizada e permitiria a realização da IATF em um momento mais preciso, resultando em melhores taxas de concepção, porém este mecanismo não foi observado neste estudo, sugerindo que ambos os indutores e suas associações parecem funcionar eficientemente em um programa de IATF, pois apesar do emprego de um único indutor afetar o DFOL e a taxa de crescimento do folículo ovulatório, estes parâmetros não impactaram sobre os índices de fertilidade.

\section{CONCLUSÃO}

Desta forma, a aplicação do benzoato de estradiol, do $\mathrm{GnRH}$ ou a associação entre estes fármacos favorece uma eficiente resposta ovulatória e satisfatória taxa de concepção em fêmeas Nelore submetidas a um protocolo de IATF. Além disso, os protocolos que utilizam o Benzoato de estradiol no momento da retirada da progesterona e o $\mathrm{GnRH}$ no momento da IA apresentam-se como uma alternativa interessante para diminuir o número de manejos sem comprometer a fertilidade. No entanto, a associação entre os fármacos não promoveu incremento nestes índices reprodutivos.

\section{REFERÊNCIAS}

ANDRADE, B. H. A.; LOIOLA, M. V. G.; CHALHOUB, M. et al. Dinâmica pré-ovulatória de vacas zebuínas usando diferentes indutores da ovulação. Congresso Brasileiro de Reprodução Animal, 19, 2011, Recife, PE, Anais... Belo Horizonte: CBRA, 2011.

AMBROSE, D. J.; RAJAMAHENDRAN, R.; KASTELIC, J. P. et al. Synchronization of ovulation and conception rates in Holstein heifers given an intravaginal progesteronereleasing device (CIDR), and estradiol cypionate, porcine LH or gonadotropin releasing hormone. Archiv Tierzucht, v.44, p.77-79,2001.

AYRES, H.; MARTINS, C. M.; FERREIRA, R. M. et al. Effect of timing of estradiol benzoate administration upon synchronization of ovulation in suckling Nelore cows (Bos indicus) treated with a progesterone-releasing intravaginal device. Animal Reproduction Science, v.109, n.1/4, p.77-87, 2008.

BARROS, C. M.; ERENO, R. L. Avanços em tratamentos hormonais para a inseminação artificial com tempo fixo (IATF) em bovinos de corte. Acta Scientiae Veterinariae, v.32, Supl., p.23-34, 2004.

BARUSELLI, P. S.; SOUZA, A. H., MARTINS, C. M. et al. Sêmen sexado: inseminação artificial e 

protocolos de indução da ovulação

transferência de embriões. Revista Brasileira de Reprodução Animal, v.31, n.3, p.374-381, 2007.

BINELLI, M.; IBIAPINA, B. T.; BISIONOTTO, R. $\mathrm{S}$. Bases fisiológicas, farmacológicas e endócrinas dos tratamentos de sincronização do crescimento folicular e da ovulação. Acta

Scientiae Veterinariae, v.34, Supl. 1, p.1-7, 2006.

BÓ, G. A.; BARUSELLI, P. S.; MARTINEZ, M. F. Pattern and manipulation of follicular development in Bos indicus cattle. Animal Reproduction Science, v.78, p.307-326, 2003.

CAVALIERI, J.; COLEMAN C.; RODRIGUES, H. et al. The effect of timing of administration of oestradiol benzoate on characteristics of oestrus, timing of ovulation and fertility in Bos indicus heifers synchronized with a progesterone releasing intravaginal insert. Autralian

Veterinary Journal, v.80, n.4, p.217-223, 2002.

CREPALDI, G. A. Eficácia de diferentes protocolos de indução da ovulação e de intervalos de inseminação em vacas de corte submetidas à IATF. 2009. 87f. Tese (Mestrado) - Universidade de São Paulo, São Paulo.

CRIVELETI JUNIOR, D. ; GOFERT, L. F. et al. Comparação entre os indutores de ovulação benzoato de estradiol e lecirelina em protocolos de inseminação artificial em tempo fixo (IATF) em novilhas Nelore pré-sincronizadas com progesterona e estrógeno. Animal

Reproduction, v.9, n.3, p.368, 2012.

CUTAIA, L.; MORENO, D.; VILLATA, L. et al. Synchrony of ovulation in beef cows treated with progesterone vaginal devices and estradiol benzoate administered at device removal or 24 hours later. Theriogenology, v.55, p.244, 2001.

FIGUEIREDO, R. A.; BARROS, C. M.; PINHEIRO, O. L. et al. Ovarian folicular dynamics in Nelore breed (Bos indicus) cattle. Theriogenology, v.47, p.1489-1505, 1997.

HENRY, M.; NEVES, J. P. Manual para Exame Andrológico e Avaliação de sêmen animal. Colégio Brasileiro de Reprodução Animal. Belo Horizonte, 1998. 49p.

HOUGHTON, P. L.; LEMENAGER, R. P. et al. Prediction of Postpartum Beef Cow Body Composition Using Weight to Height Ratio and Visual Body Condition Score. Journal of Animal Science, v.68, n.5, p.1428-1437, 1990.
INFORZATTO, G. R.; SANTOS, W. R. M.; CLIMENI, B. S. O. et al. Emprego de IATF (Inseminação Artificial em Tempo Fixo) como alternativa na reprodução da pecuária de corte. Revista científica eletrônica de medicina veterinária, v.11, p.1-8, 2008.

KIM, U- HYUNG; SUH, GUK-HYUN; NAM, HYUN-WOOK. Follicular wave emergence, luteal function and synchrony of ovulation following $\mathrm{GnRH}$ or estradiol benzoate in a CIDRtreated, lactating Holstein cows.

Theriogenology, v.63, p.260-268, 2005.

MACMILLAN, K. L.; BURKE, C. R. Effects of oestrous cycle control on reproductive efficiency. Animal Reproduction Science, v.42, p.307320, 1996.

MARTIN, L.; VETTORATO, L. F.; TAVARES, R. Z. et al. Características ultrassonográficas do corpo lúteo e do diâmetro folicular pré-ovulatório de vacas Nelore. Acta Scientiae Veterinariae, v.32,Supl., p.234, 2004.

MARTINEZ, M. F.; KASTELIC, J. P.; ADAMS, G. P. et al. The use of $\mathrm{GnRH}$ or estradiol to facilitate fixed-time insemination in an MGAbased synchronization regimen in beef cattle. Animal Reproduction Science, v.67, p.221229, 2001.

MENEGHETTI, M.; SÁ FILHO, O. G.; PERES, R. F. G. et al. Fixed-time artificial insemination with estradiol and progesterone for Bos indicus cows I: Bassis for development of protocols. Theriogenology, v.72, p.179-189, 2009.

MIZUTA, K. Estudo comparativo dos aspectos comportamentais do estro e dos teores plasmáticos de LH, FSH, progesterona e estradiol que precedem a ovulação em fêmeas bovinas Nelore (Bos taurus indicus), Angus (Bos taurus taurus) e Nelore $x$ Angus (Bos taurus x Bos taurus taurus). 2003. $98 \mathrm{f}$. Tese (doutorado) - Universidade de São Paulo, São Paulo.

MURTA, J. E. J. M. Controle farmacológico da ovulação em vacas Nelore associado acetato de melengestrol, prostaglandina e GnRH. 2011. 46f. Tese (doutorado) - Universidade Federal de Minas Gerais, Belo Horizonte.

NETO, T. M.; GOFERT, L. F.; RABESQUINI, M. $M$. et al. Comparativo entre o efeito da lecirelina e do LH como indutores de ovulação na taxa de prenhez de novilhas Nelores pré-sincronizadas com progesterona e benzoato de estradiol em um protocolo de inseminação artificial em tempo 
fixo (IATF). Animal Reproduction, v.9, n.3, p.384, 2012.

ODDE, K. G. A review of synchronization of estrus in postpartum cattle. Journal of Animal Science. v.68, p.817-830, 1990.

REIS, E. L.; GIMENES, L. U.; MARQUES, M. O. et al. Efeitos do cipionato e do Benzoato de estradiol na dinâmica folicular e luteínica de vacas Nelore. Acta Scientiae Veterinariae, v. 32 ,Supl., p.236, 2004.

ROSS, P. J.; ALLER, J. F.; CALLEJAS, S. S. et al. Estradiol benzoate given 0 or $24 \mathrm{~h}$ after the end of a progestagen treatment in postpartum suckled beef cows. Theriogenology, v.62, p.265-273, 2004.

SÁ FILHO, M.; REZENDE, C. R. L.; BISINOTTO, R. S. et al. Efeito de diferentes doses de GnRH no momento da IATF na taxa de prenhez de vacas lactantes tratadas com Norgestomet e Benzoato de estradiol. Acta scientiae Veterinariae, v. 35, Supl. 3, p.1122, 2007.

SÁ FILHO, M. F.; REIS, E. L.; VIEL JR, J. O. et al. Dinâmica folicular de vacas Nelore lactantes em anestro tratadas com progestágeno, eCG e $\mathrm{GnRH}$. Acta Scientiae Veterinariae, v.32, Supl., p.235, 2004.

\section{SAMPAIO, I. B. M. Estatística aplicada à} experimentação animal. 2. ed. Belo Horizonte: Fundação de Ensino e Pesquisa em Medicina Veterinária e Zootecnia (FEPMVZ), 1998. 221p.

SILVA , M. R.; MASSONETO, J. F.; MARINHO, D. B. et al. Efeito do tratamento com análogo de GnRH no momento da inseminação sobre a taxa de concepção em vacas Nelore primíparas submetidas ao protocolo de IATF no pantanal sul matogrossense. Acta Scientiae

Veterinariae, v.39, p.379, 2011.

SOUZA, A. H. Inseminação artificial em tempo fixo em vacas de alta produção. 2008.152f.

Tese (doutorado) - Universidade de São Paulo, São Paulo.

THOMPSON, K. E.; STEVENSON, J. S.; LAMB, G. C. et al. Follicular, hormonal and pregnancy responses of early postpartum suckled beef cows to $\mathrm{GnRH}$, Norgestomet and prostaglandin F2a. Journal Animal Scienc, v.77, p.18231832, 1999.

TORRES-JUNIOR, J. R. S.; AYRES, H.;

ARAUJO, R. L. et al. Dinâmica folicular de vacas Nelore (Bos indicus) tratadas com implante auricular de norgestomet associado ao cipionato de estradiol e submetidas a administração de GnRH 48 ou 54 horas após a retirada do implante. Acta Scientiae Veterinariae, v.35, Supl. 3, p.1111, 2007. 